\title{
Wavelet p-Leader Non-Gaussian Multiscale Expansions for EEG series: an Exploratory Study on Cold-Pressor Test
}

\author{
V. Catrambone ${ }^{* 1}$, G. Valenza ${ }^{1}$, E. P. Scilingo ${ }^{1}$, N. Vanello ${ }^{1}$, H. Wendt $^{2}$, R. Barbieri ${ }^{3}$, and P. Abry ${ }^{4}$
}

\begin{abstract}
Brain dynamics recorded through electroencephalography (EEG) have been proven to be the output of a nonstationary and nonlinear system. Thus, multifractality of EEG series has been exploited as a useful tool for a neurophysiological characterization in health and disease. However, the role of EEG multifractality under peripheral stress is unknown. In this study, we propose to make use of a novel tool, the recently defined non-Gaussian multiscale analysis, to investigate brain dynamics in the range of $4-8 \mathrm{~Hz}$ following a cold-pressor test versus a resting state. The method builds on the wavelet p-leader multifractal spectrum to quantify different types of departure from Gaussian and linear properties, and is compared here to standard linear descriptive indices. Results suggest that the proposed non-Gaussian multiscale indices were able to detect expected changes over the somatosensory and premotor cortices, over regions different from those detected by linear analyses. They further indicate that preferred responses for the contralateral somatosensory cortex occur at scales $2.5 \mathrm{~s}$ and $5 \mathrm{~s}$. These findings contribute to the characterization of the so-called central autonomic network, linking dynamical changes at a peripheral and a central nervous system levels.
\end{abstract}

\section{INTRODUCTION}

Scale-free dynamics in EEG. Electroencephalographic (EEG) signals have been proven to be the output of a highly nonlinear system for which scale-free dynamics have been frequently reported in the literature, historically evidenced by $1 / f^{\beta}$ power law (2nd order) spectra [1], [2]. Several different models have been proposed to quantify the mechanisms linking different time scales for scale-free temporal dynamics. A simple, elegant model is given by self-similarity, modelling power laws at all statistical orders with exponents solely controlled by one single parameter $H$, the so-named selfsimilarity index, or Hurst exponent (evidenced on spectra, $\beta=2 H+1)$. Self-similarity in EEG series has been demonstrated for oscillations at different frequency bands at rest and/or task conditions [2]. Experimental evidences of scale-free brain network activation has also been found in other brain-related series including the ones from functional magnetic resonance imaging, electrocorticography, and magnetoencephalography [3]-[5].

Multifractal modeling. Yet, EEG series have also been shown to display fluctuating local singular behaviours, beyond simple self-similarity with single exponent $H$ and

\footnotetext{
1 Bioengineering and Robotics Research Center E.Piaggio \& Dipartimento di Ingegneria dell'Informazione, Scuola di Ingegneria, University of Pisa, Italy;

2 IRIT, CNRS, Université de Toulouse, France;

3 Department of Electronics, Informatics and Bioengineering, Politecnico di Milano, Italy;

${ }^{4}$ Université de Lyon, ENS de Lyon, Université Claude Bernard, CNRS, Laboratoire de Physique, Lyon, France;

* Corresponding author. Email at: vincenzo.catrambone@ing.unipi.it
}

requiring a model with a whole collection of exponents $H=h(t)$ to comprehensively characterize the temporal dynamics [6], [7]. Mathematically, such a model can be efficiently framed using multifractal (MF) analysis, in form of the multifractal spectrum $D(h)$, and can quantify transient and local non-Gaussian structures that cannot be described by a single parameter $H$. In EEG series, MF properties have been evidenced in several experimental conditions, including different sleep stages [8], [9], motor imagery and real visualmotor tasks [10], mental arithmetics [11], epileptic seizures [12], being also exploited for brain-computer interface applications [13]. Several different practical analysis tools have been proposed to study the MF properties of real-world data, including the wavelet transform modulus maxima method (WTMM) [14], and the MF detrended fluctuation analysis (MF-DFA) [15]. Current state of the art MF analyses rely on discrete wavelet transforms and are performed using the so-called wavelet leader multifractal formalisms [16] and its generalization using p-leaders [17].

Multiscale non-Gaussian expansions. It has recently been shown that this wavelet p-leader MF formalism provides novel nonlinear indices of physiological dynamics scaling properties that were not assessed previously [18]. Moreover, elaborating on the underlying MF model, the definition of versatile multiscale non-Gaussian expansion coefficients has been proposed for wavelet p-leaders. These indices are specifically designed to quantify non-Gaussian dynamics in data, thus enabling to discern two signals that share linear and Gaussian features and power spectra. In addition, while these quantities inherit the key beneficial statistical properties of p-leaders, they do not require strict power law models, hence lend extra flexibility [18].

Goals and contributions. The goal of this paper is to investigate the use of these novel nonlinear multiscale expansions for the characterization of brain responses to peripheral stimuli. Specifically, we investigate non-Gaussian multiscale properties of EEG series during sympathetic stress and, consequently, baroreflex activation as the cold-pressor test (CPT) [19]-[21]. Previous studies investigating EEG changes following a CPT elicitation mostly focused on linear properties [19], [20], and MF and multiscale non-Gaussian changes in this regard are unknown. Here, we focus on EEG oscillations within the $\theta$ band $(4-8 \mathrm{~Hz})$, exploiting a dataset of 24 right-handed healthy volunteers undergoing to resting and CPT sessions. Our results indicate that multiscale nonGaussian indices reveal changes in cortical regions involved in the experiment to which linear analyses are blind. 


\section{Materials And Methods}

\section{A. Experimental Setup and EEG Data Collection}

Thirty right-handed healthy volunteers (15 females, 26.7 years on average) were enrolled in the study after giving their informed consent. Data from six participants were rejected due to significant artefacts in EEG recordings. The experimental timeline consisted of a period of $3 \mathrm{~min}$ resting state, followed by a CPT session during which the experimentalist submerged the subject's non-dominant hand, up to the wrist, in a solution of ice and water. The temperature of the solution was maintained between 0 and $4{ }^{\circ} \mathrm{C}$. Subjects were asked not to move their hand for up to $3 \mathrm{~min}$, as per the average time threshold in healthy people before feeling pain [22]. In case of early pain, volunteers were free to terminate the CPT and move to the next experimental session. The experimental procedures were approved by the local ethical committee. Further details maybe found in [21].

Throughout the protocol, a set of physiological signals were gathered, including high-resolution 128 channels EEG sampled at $500 \mathrm{~Hz}$. To minimize subject movements and hemodynamic stabilisation, participants were asked to sit comfortably on a chair throughout the recording.

\section{B. Data Preprocessing}

EEG preprocessing was performed following the HAPPE procedure, which is fully described in [23]. Briefly, a subset of signals from 90 electrodes was selected and high-pass filtered at $1 \mathrm{~Hz}$. Electrical noise at $50 \mathrm{~Hz}$ was removed through a multi-taper regression approach, and bad channels were identified and removed based on eventual high impedances or belonging to the $1 \%$ tails of the EEG data distribution, calculated by the normed joint probability of the mean $\log$ power from 1 to $125 \mathrm{~Hz}$.

The preprocessing pipeline also foresaw a waveletenhanced independent component analysis (W-ICA) followed by a further ICA step to eventually reject additional artefact components. While the first W-ICA step removed some of the most severe artefacts, including high-amplitude artefacts (e.g., blinks), and discontinuities in the recording, the subsequent ICA with an automated component rejection was especially effective at detecting and removing muscle artefact components [23]. At this point, channels removed during the bad channel rejection step were interpolated through a spherical algorithm. The final processing step comprised a re-referencing of the EEG data using average values across all channels.

Below, we report on the investigation of the dynamical properties of the time-varying power of the spectrum calculated within the $\theta$ band of the EEG (i.e., within $[4,8] \mathrm{Hz}$ ) only. For each subject, the time-varying power was calculated on EEG series from each electrode by applying a Hamming window and using a modified periodogram approach, with time windows of 2 seconds overlapped by $50 \%$, thus a $1 \mathrm{~s}$ resolution.

\section{Multifractals and Non-Gaussian Multiscale Analyses}

1) Discrete wavelet transform: The discrete wavelet transform coefficients are obtained as the inner products of the matrix $X$ and the orthonormal collection of functions $\psi_{j, k}(t)=2^{-j} \psi\left(2^{-j} t-k\right)_{(j, k) \in \mathbb{N}^{2}}$, constructed by dilatations to scales $j$ and translations to position $2^{j} k$ from a mother wavelet $\psi: d_{X}(j, k)=\left\langle\psi_{j, k} \mid X\right\rangle$, a specific oscillatory reference pattern with narrow time and frequency support (cf. [24]).

For self-similar models, the wavelet spectrum

$$
S_{d_{X}}(j, q=2)=\frac{1}{n_{j}} \sum_{k=1}^{n_{j}}\left|d_{X}(j, k)\right|^{q} \simeq K 2^{j q H},
$$

displays power laws with exponent controlled by the Hurst parameter $H$ which enables its estimation via log-log plot regressions [25] (here, $n_{j}$ is the number of $d_{X}(j, k)$ available at scale $2^{j}$ ). The wavelet spectrum can be directly related to the Fourier spectrum, and $H$ to linear data properties and energy distribution across frequency bands [25], [26].

2) Wavelet p-Leaders and Multifractality: Multifractal models enrich self-similarity in that $H$ is replaced by an entire interval of local self-similarity exponents $H=h(t)$ that are assessed by the multifractal spectrum $D(h)$, which quantifies the temporal repartition of these exponents [16], [26]. To estimated $D(h)$ from data, one must replace $q=2$ in (1) with a range of positive and negative moments $q$, and wavelet coefficients with wavelet p-leaders $\ell_{X}^{(p)}$.

They are defined as local $\ell^{p}$ norms of wavelet coefficients in a narrow temporal neighbourhood over all finer scales

$$
\ell_{X}^{(p)}(j, k)=\left(2^{j} \sum_{\lambda^{\prime} \subset 3 \lambda_{j, k}} 2^{-j^{\prime}}\left|d_{X}\left(\lambda^{\prime}\right)\right|^{p}\right)^{1 / p}
$$

with $\lambda_{j, k}=\left[k 2^{j},(k+1) 2^{j}\right)$ and $3 \lambda_{j, k}=\bigcup_{m\{-1,0,1\}} \lambda_{j, k+m}$. It can be shown that the cumulants of log-leaders

$$
C_{m}^{(p)}(j) \equiv \operatorname{Cum}_{m} \log \left(\ell_{X}^{(p)}(j)\right) \simeq c_{m}^{0}+c_{m} \log \left(2^{j}\right)
$$

enable constructing estimates for the multifractal spectrum $D(h): c_{1}$ quantifies the mode of $D(h)$ and linear data properties that are closely related to $H$, while $c_{2}, c_{3}, c_{4}$ are nonlinear features that quantify width, asymmetry and kurtosis of the multifractal spectrum, respectively (see e.g. [16] and references therein for details on multifractal analysis).

3) Non-Gaussian Multiscale Representation: Assessing higher-order cumulants as in (3) can be problematic for real data. Instead, the use of a multiscale non-Gaussian expansion

$$
L_{q}^{2 P}(j)=\sum_{i=1}^{P} \frac{\log \left(S_{\ell_{X}}\left(j, q_{2 i-1}\right)\right)}{q_{2 i-1}}-\frac{\log \left(S_{\ell_{X}}\left(j, q_{2 i}\right)\right)}{q_{2 i}}
$$

has recently been proposed in [18], where $S_{\ell_{X}}\left(j, q_{i}\right)=$ $\frac{1}{n_{j}} \sum_{k=1}^{n_{j}} \ell_{X}^{(p)}(j, k)^{q_{i}}$. It can be shown that the coefficients $L_{q}^{2 P}(j)$ allow to probe higher-order cumulants, and thus nonGaussian properties, using moments $q_{i}$ of low order only:

$$
L_{q}^{2 P}(j)=\sum_{m=2}^{\infty} C_{m}(j) \frac{\sum_{i=1}^{P} q_{2 i-1}^{m-1}-q_{2 i}^{m-1}}{m !} .
$$

Since $C_{1}(j)$ does not appear in (5), $L_{q}^{2 P}(j)$ quantifies only the nonlinear data properties. Moreover, tuning $q_{i}$ enables us to discern different natures of departure from Gaussian, see [18] for details. 


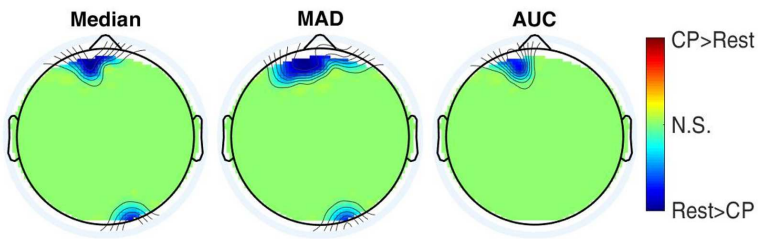

Fig. 1: Linear features. p-value topographic maps from nonparametric Wilcoxon tests between resting state and CPT elicitation for standard EEG features (i.e. median, Maximum Absolute Deviation, and Area Under Curve) calculated on the time-varying power in the $\theta$ band: blue (red) areas indicate significant differences $(p \leq 0.05)$ with higher (lower) values during the resting state with respect to CPT session (green areas: no statistical differences between sessions, $p>0.05$ ).

\section{Statistical Comparison}

For each time-varying $\theta$ band power spectrum series, features from a non-Gaussian multiscale representation, as well as from standard analysis were statistically compared between the two experimental conditions, the resting and CPT session. The features from standard analysis included the median, the maximum absolute deviation (MAD), and the area under the curve (AUC) of the time-varying power in the $\theta$ band. Statistical comparison between sessions was performed using non-parametric Wilcoxon tests for paired samples, and p-values were adjusted for multiple comparison through a permutation test, with a total of 1000 permutations.

\section{EXPERIMENTAL RESUlts}

Experimental results comprise the statistical comparison between resting and CPT sessions for features from a standard analysis and for non-Gaussian multiscale expansions, and are shown as p-value topographic maps.

\section{A. Standard Analysis}

Fig. 1 shows three p-value topographic maps from the non-parametric Wilcoxon tests for the median, MAD, and the integral of the time-varying $\theta$ power. Few significant differences are found in the frontal and occipital lobes, with decreasing trends during the CPT session with respect to the preceding resting session. This is in agreement with previous findings on CPT [19], [20], as well as further PET/fMRI investigations [27], [28]; it has been suggested that these changes may represent the inhibition of sensorial perception after the nociceptive input [19].

\section{B. Non-Gaussian Multiscale Analysis}

The nonlinear analysis was performed using a Daubechies wavelet, with $N_{\phi}=3$ vanishing moments, and three different non-Gaussian multiscale expansion coefficients $L_{q}^{2 P}(s)$, defined in Table I, and quantifying different aspects of departure from Gaussianity.

Fig. 2 shows p-value topographic maps from the nonparametric Wilcoxon test for non-Gaussian indices LQ1, LQ2 and LQ3 over 5 different time scales corresponding to $2.5,5,10,21$, and 42 seconds. The scales listed above refer to the oscillation during time of the signal derived from the
TABLE I: Non-Gaussian expansion indices

\begin{tabular}{r|cr}
\hline & moments $q_{i}$ & cumulants $C_{m}$ active in (5) \\
\hline LQ1 & $(0.25,2)$ & $m \geq 2$ \\
LQ2 & $(-2,2)$ & any departure from Gaussian \\
& & $m=2,4, \ldots$ \\
LQ3 & $(0.25,0.75,2.5,2))$ & symmetric properties \\
& & $m \geq 3$ \\
& & non log-normal non-Gaussian \\
\hline
\end{tabular}

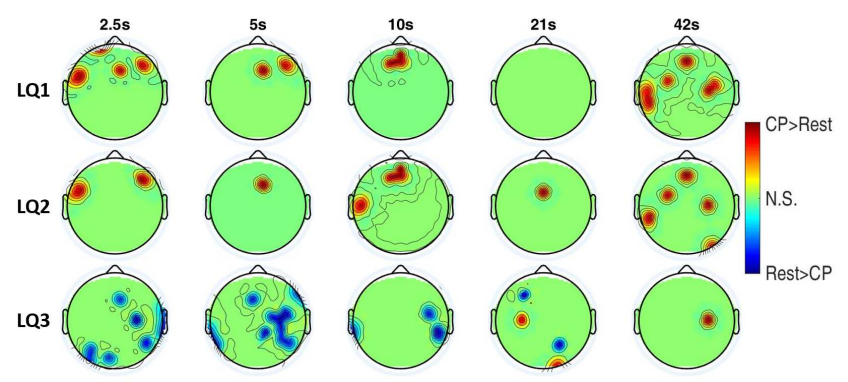

Fig. 2: Non-Gaussian multiscale expansion. p-value topographic maps from non-parametric Wilcoxon tests between resting state and CPT elicitation for Non-Gaussian multifractal indices calculated on the time-varying power in the $\theta$ band: blue (red) areas indicate significant differences $(p \leq 0.05)$ with higher (lower) values during the resting state with respect to CPT session (green areas: $p>0.05$ ).

power in the $\theta$ band, they must not to be confused with correspondent oscillations in the EEG signals. Here, LQ1 quantifies any departure from Gaussian, LQ2 only quantifies the symmetric portions of departure from Gaussian, and LQ3 non log-Normal type departures. Significant differences are associated with LQ1 and LQ2 over the midline frontal brain areas at all scales, as well as prefrontal regions at $2.5 \mathrm{~s}$ and $5 \mathrm{~s}$ and, at coarser scales, over the left temporal lobe and right primary motor cortex. At a $21 \mathrm{~s}$ scale, no significant changes were found for LQ1 and only one over the central prefrontal cortex for LQ2. On LQ3, significant differences were found over the right somatosensory cortex at all scales, as well as over the left occipital cortex at $2.5 \mathrm{~s}$ and left temporal cortex at $5 \mathrm{~s}$ and 10s. As expected, the three non Gaussian indices show different behaviour across the scalp. As a matter of fact, LQ1 and LQ2 share many significant electrodes, while LQ3 highlights separated regions, thus evidencing the log-normal characterization of the $\theta$ power time series in the regions enhanced by LQ1 and LQ2, while the non-Gaussinity must have a different nature in regions significant in LQ3.

These results show that the nonlinear non-Gaussian indices are able to detect, over several time scales, significant changes in brain regions that were expected to be involved in this protocol and for which linear features show no significant differences, including somatosensory and premotor cortices [29]. Interestingly, these significant changes in nonlinear features are in agreement with our previous findings related to brain-heart information transfer during CPT [21], [30], especially referring to right latero-temporal and right dorso-parietal cortices, as shown for LQ3. 


\section{DisCUSSION AND CONCLUSION}

We investigated non-Gaussian multiscale indices of EEG dynamics during a peripheral elicitation as CPT with respect to resting conditions. To the best of our knowledge, such EEG multifractal-inspired nonlinear multiscale analysis has not been applied to investigate changes occurring following a CPT, especially considering the recently proposed nonGaussian multiscale representation from wavelet p-leaders.

We showed that the nonlinear non-Gaussian multiscale coefficients detected significant differences in brain regions that were expected to be involved in this protocol, that a standard linear analysis was not able to detect. In fact, standard analysis on $\theta$ band power using median, MAD, and AUC indices (Figure 1) shows significant changes only over the midline frontal region, potentially related to nociceptive perception [19].

Our results therefore suggest that several brain activity changes in the $\theta$ band during a sympathetic stressor are related to the nonlinear non-Gaussian MF properties of the neural dynamics per se. Furthermore, results indicate that preferred responses for the contralateral somatosensory cortex occur at scales $2.5 \mathrm{~s}$ and $5 \mathrm{~s}$. Although at a speculative level, we argue that the activity of superior parietal lobe, known to be critical for sensorimotor integration [31], results in a non-Gaussian dynamics detectable through LQ3 (nonlognormal shape). Also, activations detectable through a linear and a nonlinear analysis over the midline frontal cortex and premotor cortex maybe related to a motor control task, which might be due to the control that subjects had to exert over their hand in order to maintain the experimental posture.

Our findings may also help for the investigation of the functional links between peripheral and the central nervous systems, especially considering the Central Autonomic Network [32], particularly in those related to brain-heart information transfer during CPT [21].

Main limitations of our work are related to the fact we accounted for the EEG $\theta$ band exclusively; other EEG frequencies will be investigated in future studies. In conclusion, although we are still far from a clear neurophysiological explanation for EEG non-Gaussian and multifractal changes, this study support the evidence that neural dynamics may act in a non-Gaussian, multifractal fashion.

\section{REFERENCES}

[1] D. Van de Ville et al., "Eeg microstate sequences in healthy humans at rest reveal scale-free dynamics," Proceedings of the National Academy of Sciences, p. 201007841, 2010.

[2] C. Stam et al., "Scale-free dynamics of global functional connectivity in the human brain," Human brain mapping, vol. 22, no. 2, pp. 97-109, 2004.

[3] D. La Rocca et al., "Self-similarity and multifractality in human brain activity: A wavelet-based analysis of scale-free brain dynamics," Journal of Neuroscience Methods, 2018. In press.

[4] C. Gentili et al., "Proneness to social anxiety modulates neural complexity in the absence of exposure: a resting state fmri study using hurst exponent," Psychiatry Research: Neuroimaging, vol. 232, no. 2, pp. 135-144, 2015.

[5] C. Stam, "Nonlinear dynamical analysis of EEG and meg: review of an emerging field," Clinical neurophysiology, vol. 116, no. 10, pp. 22662301, 2005.

[6] T. s Vicsek, Fractal growth phenomena. World scientific, 1992.
[7] H. Takayasu, Fractals in the physical sciences. Manchester University Press, 1990.

[8] B. Weiss et al., "Spatio-temporal analysis of monofractal and multifractal properties of the human sleep EEG," journal of neuroscience methods, vol. 185, no. 1, pp. 116-124, 2009.

[9] I. Song et al., "Multifractal analysis of sleep EEG dynamics in humans," in Neural Engineering, 2007. CNE'07. 3rd IEEE/EMBS, pp. 546-549, IEEE, 2007.

[10] D. Popivanov et al., "Multifractality of decomposed EEG during imaginary and real visual-motor tracking," Biological Cybernetics, vol. 94, no. 2, pp. 149-156, 2006.

[11] Q. Wang et al., "Real-time mental arithmetic task recognition from EEG signals," IEEE Transactions on Neural Systems and Rehabilitation Engineering, vol. 21, no. 2, pp. 225-232, 2013.

[12] Y. Zhang et al., "Multifractal analysis and relevance vector machinebased automatic seizure detection in intracranial EEG," International journal of neural systems, vol. 25, no. 06, p. 1550020, 2015.

[13] N. Brodu et al., "Exploring two novel features for EEG-based braincomputer interfaces: Multifractal cumulants and predictive complexity," Neurocomputing, vol. 79, pp. 87-94, 2012.

[14] J. Muzy et al., "Multifractal formalism for fractal signals: The structure-function approach versus the wavelet-transform modulusmaxima method," Physical review E, vol. 47, no. 2, p. 875, 1993.

[15] J. Kantelhardt et al., "Multifractal detrended fluctuation analysis of nonstationary time series," Physica A: Statistical Mechanics and its Applications, vol. 316, no. 1-4, pp. 87-114, 2002.

[16] H. Wendt et al., "Bootstrap for empirical multifractal analysis," IEEE signal processing magazine, vol. 24, no. 4, pp. 38-48, 2007.

[17] S. Jaffard et al., "p-exponent and p-leaders, part i: Negative pointwise regularity," Physica A: Statistical Mechanics and its Applications, vol. 448, pp. 300-318, 2016.

[18] H. Wendt et al., "Wavelet $p$-leader non gaussian multiscale expansions for heart rate variability analysis in congestive heart failure patients," IEEE Transactions on Biomedical Engineering, vol. 66, no. 1, pp. 8088, 2019.

[19] S. Ferracuti et al., "Quantitative EEG modifications during the cold water pressor test: hemispheric and hand differences," International Journal of Psychophysiology, vol. 17, no. 3, pp. 261-268, 1994.

[20] P. Chang et al., "Dynamic changes and spatial correlation of EEG activities during cold pressor test in man," Brain research bulletin, vol. 57, no. 5, pp. 667-675, 2002.

[21] V. Catrambone et al., "Time-resolved directional brainheart interplay measurement through synthetic data generation models," Annals of Biomedical Engineering, 2019.

[22] J. Cui et al., "Baroreflex modulation of muscle sympathetic nerve activity during cold pressor test in humans," Am. J. of Phys, vol. 282, no. 5, pp. H1717-H1723, 2002.

[23] L. Gabard-Durnam et al., "The harvard automated processing pipeline for electroencephalography (happe): standardized processing software for developmental and high-artifact data," Frontiers in neuroscience, vol. 12, p. 97, 2018.

[24] S. Mallat, A wavelet tour of signal processing. Elsevier, 1999.

[25] D. Veitch et al., "A wavelet-based joint estimator of the parameters of long-range dependence," IEEE Transactions on Information Theory, vol. 45, no. 3, pp. 878-897, 1999.

[26] M. Doret et al., "Multifractal analysis of fetal heart rate variability in fetuses with and without severe acidosis during labor," American journal of perinatology, vol. 28, no. 04, pp. 259-266, 2011.

[27] K. Casey, "Forebrain mechanisms of nociception and pain: analysis through imaging," Proceedings of the National Academy of Sciences, vol. 96, no. 14, pp. 7668-7674, 1999.

[28] U. Frankenstein et al., "Distraction modulates anterior cingulate gyrus activations during the cold pressor test," Neuroimage, vol. 14, no. 4, pp. 827-836, 2001.

[29] R. Coghill et al., "Pain intensity processing within the human brain: a bilateral, distributed mechanism," Journal of neurophysiology, vol. 82, no. 4, pp. 1934-1943, 1999.

[30] V. Catrambone et al., "A new modelling framework to study timevarying directional brain-heart interactions: Preliminary evaluations and perspectives," in IEEE EMBC, pp. 4611-4614, IEEE, 2018.

[31] D. Wolpert et al., "Maintaining internal representations: the role of the human superior parietal lobe," Nature neuroscience, vol. 1, no. 6, p. 529, 1998.

[32] F. Beissner et al., "The autonomic brain: an activation likelihood estimation meta-analysis for central processing of autonomic function," Journal of Neuroscience, vol. 33, no. 25, pp. 10503-10511, 2013. 